\title{
Impacto do Treinamento no Trabalho: Investigando Variáveis Motivacionais e Organizacionais como suas Preditoras
}

\author{
Érika Rodrigues Magalhães Lacerda \\ Gardênia Abbad
}

\section{Resumo}

Essa pesquisa teve como objetivo principal testar um modelo de avaliação de Impacto do Treinamento no Trabalho. O estudo foi desenvolvido em três etapas: (1) construção e validação da escala de Valor Instrumental do Treinamento; (2) aperfeiçoamento e validação das escalas Motivação para Aprender, Motivação para Transferir e Suporte Pré-Treinamento; (3) investigação do relacionamento entre características dos participantes dos treinamentos (Motivação para Aprender e para Transferir e Valor Instrumental), Reações e Suporte à Transferência, como variáveis antecedentes, e Impacto do Treinamento no Trabalho, como variável critério. As respostas dos participantes de 28 cursos às escalas foram submetidas a análises exploratórias, análise dos componentes principais, análise fatorial e de consistência interna, além de análises de regressão múltipla padrão. Os resultados das etapas 1 e 2 mostraram escalas unifatoriais psicometricamente válidas e precisas. Na etapa 3, a análise de regressão múltipla padrão demonstrou apenas três variáveis explicativas de auto-avaliação de Impacto do Treinamento no Trabalho: Suporte Psicossocial, Valor Instrumental do Treinamento e Reação do Participante ao Instrutor. São discutidas algumas implicações teóricas e práticas desses achados.

Palavras-chaves: impacto do treinamento; características da clientela; motivação; valor instrumental do treinamento; suporte psicossocial.

\begin{abstract}
This research had as a main objective to test a model of Training Impact Evaluation at Work. Three stages were accomplished: (1) the construction and validation of a scale of Training Instrumental Value; (2) the improvement and validation of the scales of Motivation to Learn, Motivation to Transfer and Support Before Training; and (3) the investigation of the relationship among the characteristics of the training participants (Motivation to Learn, Motivation to Transfer and Training Instrumental Value), Reactions and Support to the Transfer of Training; as independent variables, and Impact of Training at Work, as dependent variable. The answers of the participants of 28 courses to the scales were submitted to exploratory data analysis, principal components analysis, factor analysis, reliability analysis and standard multiple regression. The results of the stages 1 and 2 showed valid and accurate one factor scales. In the stage 3, the standard multiple regression analysis showed only three explained variable of self-evaluation of Impact of Training at Work: Manager and Social Support, Training Instrumental Value and Reaction of the Participant to the Instructor. Some theoretical and practical implications of those discoveries are discussed.
\end{abstract}

Key words: training impact; characteristics of the training participants; motivation; training instrumental value; manager and social support. 


\section{INTRODUÇÃO}

As organizações têm questionado a eficácia das ações de treinamento, buscando avaliar o retorno de seus investimentos nessa área. No entanto, o uso do treinamento é uma importante alternativa para a mudança de atitudes, conhecimentos ou habilidades necessárias ao desempenho adequado do capital humano na empresa.

Há, nas empresas, poucos estudos demonstrando resultados palpáveis das ações de treinamento, as quais, muitas vezes, são compradas como pacotes e aplicadas sem estudo sistemático de necessidades reais de treinamento. Tais pacotes evidenciam modismos que prometem revolucionar a produtividade nas áreas e a motivação dos funcionários, mas não demonstram confirmação empírica da efetividade de suas ações. Borges-Andrade e Abbad (1996) apontaram os riscos associados às políticas de importação de pacotes de treinamento, já que o Brasil possui suas próprias especificidades culturais e requer a introdução de novas tecnologias, a elaboração de treinamentos eficazes e de linhas de pesquisa que facilitem o desenvolvimento social e econômico brasileiro.

Há avanços nas pesquisas sobre treinamento. Um deles diz respeito à identificação de fatores que afetam a eficácia de treinamentos em ambientes organizacionais. No entanto, são poucas as evidências de que as organizações estejam realmente aplicando os resultados de pesquisas científicas na elaboração de seus programas de treinamento. Salas e Cannon-Bowers (2001) acreditam na chegada de uma nova era na área de Treinamento e Desenvolvimento, na qual haveria maiores trocas entre pesquisas empíricas e sua aplicação nas organizações $^{(1)}$.

\section{Definições}

Goldstein (1991) definiu treinamento como uma aquisição sistemática de atitudes, conceitos, conhecimentos, regras ou habilidades que resultem na melhoria do desempenho no trabalho, obtidos por meio da análise de tarefas e princípios da tecnologia instrucional. Para Wexley (1984) treinamento é visto como um esforço planejado de uma organização para facilitar a aprendizagem de comportamentos exigidos pelo trabalho. 
O treinamento é apenas uma das intervenções que afetam desempenho no trabalho. É, portanto, somente uma das tentativas formais de garantir a aquisição de novos conhecimentos, habilidades e maior compreensão da tarefa, não afetando, necessariamente, condições organizacionais (materiais e sociais) relacionadas ao desempenho.

A teoria de sistemas tem sido utilizada para a compreensão do processo de treinamento. Borges-Andrade (1982) entende treinamento como um conjunto de atividades interdependentes ou como um subsistema organizacional, no qual o levantamento das necessidades de treinamento fornece informações que viabilizam o planejamento, que, por sua vez, orientará a execução e a avaliação do evento instrucional. Este último fornecerá dados para a avaliação, que retroalimentará os demais componentes do sistema, sugerindo ajustes em cada um deles.

Os pesquisadores concordam que a avaliação é uma importante parte do sistema de treinamento. Segundo Goldstein (1991), avaliação de treinamento é uma coleta sistemática de informações descritivas e valorativas necessárias para tornar eficazes as decisões relacionadas à seleção, adoção, valorização e modificação de várias atividades instrucionais.

\section{Modelos de Avaliação e Variáveis Explicativas de Resultados de Treinamento}

Vários são os níveis de critérios de avaliação encontrados na literatura. Em geral, os modelos abordam três níveis de avaliação: (1) Reação; (2) Aprendizagem; e (3) Impacto do Treinamento no Trabalho, Comportamento no Cargo e/ou Transferência.

Reação, conforme Abbad, Gama e Borges-Andrade (2000, p.26) é compreendida como "o nível de satisfação dos participantes com a programação, o apoio ao desenvolvimento do curso, a aplicabilidade, a utilidade e os resultados do treinamento".

Aprendizagem, de acordo com Abbad (1999, p.38), "refere-se ao grau de assimilação e retenção dos conteúdos ensinados no curso, medido em termos dos escores obtidos pelo participante em testes ou provas de conhecimentos aplicados pelo instrutor ao final do curso”. Esse nível não será enfocado no presente trabalho por duas razões principais: (1) intenção de propor um modelo mais parcimonioso de avaliação de resultados de treinamento; e (2) tempo escasso para elaborar, validar, aplicar e avaliar pré e pós-testes na organização estudada.

Ainda de acordo com Abbad (1999), Impacto do Treinamento no Trabalho 
compreende o conceito de transferência de treinamento, que se refere à aplicação correta, no ambiente de trabalho, de conhecimentos, habilidades ou atitudes adquiridas em situações de treinamento.

Hamblin (1978) adaptou o modelo de Kirkpatrick (1976), composto de quatro níveis (Reação, Aprendizagem, Comportamento no Cargo e Resultados), desdobrando o quarto nível em dois outros: Mudança Organizacional e Valor Final; ele concebeu também esses níveis como seqüenciais, lineares e correlacionados entre si.

Alliger e Janak (1989) criticaram o modelo de critérios de avaliação de treinamento utilizado por Kirkpatrick (1976), afirmando que os relacionamentos entre os níveis de avaliação tendem a ser fracamente correlacionados entre si. Esses autores basearam-se nos três pressupostos de Kirkpatrick (1976) para tecer suas críticas.

No Brasil, até 1996, o nível de avaliação mais relatado nos artigos era o de aprendizagem. Esse quadro mudou. A última revisão de Borges-Andrade (2000) sobre medidas demonstra que outros estudos começaram a enfocar o terceiro nível de avaliação integrado ao segundo e, em alguns casos, ao primeiro nível.

Os modelos de Kirkpatrick (1976) e Hamblin (1978) não têm possibilitado o entendimento das relações entre os níveis e as variáveis que os afetam. Modelos de avaliação de sistemas instrucionais mais abrangentes passaram a incluir, além dos critérios de eficácia, variáveis relacionadas ao ambiente, às clientelas e aos procedimentos instrucionais.

Entre estes modelos, destaca-se o de Borges-Andrade (1982), que desenvolveu o Modelo de Avaliação Integrado e Somativo de Sistemas Instrucionais (MAIS), e o de Abbad (1999), que construiu o Modelo Integrado de Avaliação de Impacto do Treinamento no Trabalho (IMPACT), inspirado principalmente no modelo MAIS de Borges-Andrade (1982).

Abbad (1999) identificou que as principais variáveis preditoras de Impacto do Treinamento no Trabalho pertenciam aos componentes Suporte à Transferência e Reações. Além disso, entre as variáveis do componente Características da Clientela, mereceram destaque na pesquisa as variáveis motivacionais, as quais explicaram uma porção significativa de quase todas as variáveis critério estudadas. A autora salienta que as "relações entre Reação, Aprendizagem e Impacto não são conclusivas, havendo, ainda, poucas tentativas de identificação das variáveis preditoras de cada um desses níveis de avaliação” (Abbad, 1999, p.37). 


\section{Reação e Impacto do Treinamento no Trabalho}

O conceito de Reação, presente nos modelos tradicionais de Kirkpatrick (1976) e Hamblin (1978), tem sofrido a inclusão de outras variáveis. Warr e Bunce (1995) demonstraram três medidas de Reação fatorialmente distintas: satisfação com o treinamento, percepção de utilidade e de dificuldade do curso. Alliger et al. (1997) concordaram com essa posição, afirmando ser importante distinguir as reações afetivas de um programa de treinamento das reações de utilidade, por exemplo. Assim propuseram dois componentes básicos para Reação: o afetivo (satisfação) e o cognitivo (percepções de utilidade e dificuldade do curso).

Abbad, Pantoja e Pilati (2001) criticaram a ampliação e a revisão do conceito, afirmando que incluir percepção de dificuldade na definição de Reação poderia levar a confusões conceituais e metodológicas na área. Essas ampliações conceituais "podem fragilizar as medidas de Reação e Aprendizagem, pois, ao invés de mensurarem grau de dificuldade por meio de testes de aprendizagem, sugerem o uso de avaliações perceptuais [para fazê-lo]" (Abbad, Pantoja e Pilati, 2001, p.9). Neste trabalho, Reação refere-se à satisfação dos participantes com a programação do curso e com o desempenho do instrutor, bem como a utilidade e resultados percebidos no treinamento.

Abbad, Gama e Borges-Andrade (2000) observaram que medidas válidas e confiáveis de Reação ainda são raras na literatura especializada. Esses autores obtiveram duas escalas: Reações ao Programa de Treinamento e ao Desempenho do Instrutor. Ao contrário de Alliger et al. (1997), eles encontraram forte correlação entre Reações e Impacto do Treinamento no Trabalho, mas fraca com Aprendizagem.

Já na área de Impacto do Treinamento no Trabalho, Abbad (1999) ressalta que uma das questões empíricas de maior interesse nesse nível refere-se ao efeito de eventos instrucionais e sua relação com Características da Clientela e Ambiente Organizacional sobre os níveis de desempenho no trabalho. Essa autora também informa que a maioria dos estudos que trabalha com essa variável é correlacional de campo e atualmente utiliza modelos multivariados de investigação.

No presente estudo, Impacto do Treinamento no Trabalho refere-se à autoavaliação dos efeitos genéricos do treinamento sobre o desempenho e as atitudes do treinando. Trata-se do terceiro nível de avaliação do modelo de Hamblin (1978), definido como impacto em amplitude, por gerar informações sobre efeitos gerais do treinamento no comportamento do participante, não relacionados especificamente aos objetivos instrucionais do curso. 


\section{Características Individuais dos Participantes}

Algumas variáveis são importantes determinantes dos resultados de treinamentos, entre elas, as características individuais dos treinandos. A linha de pesquisa sobre a clientela caracteriza-se por investigar o relacionamento entre características pessoais (demográficas, cognitivas, afetivas, motivacionais) e a eficácia de eventos instrucionais, em termos de Aprendizagem, Reação e Transferência (Abbad, 1999). Neste estudo, foram abordados os construtos de Motivação para Aprender, Motivação para Transferir (a aprendizagem) e Valor Instrumental do Treinamento.

O conceito de motivação, como precondição de treinamento e desenvolvimento, possui uma série de abordagens e necessita de maior clareza para cada uma de suas definições. Abbad, Pantoja e Pilati (2001) observaram que há pouco esforço para eliminar confusões conceituais nos estudos sobre motivação, afirmando que essa variável é tratada tanto como causa quanto como razão ou justificativa para as ações dos indivíduos. São várias as teorias de motivação voltadas para o treinamento e a literatura demonstra pequenos avanços teóricos.

Motivação para Treinar ou Motivação para Aprender pode ser definida como direção, esforço, intensidade e persistência com que os treinandos se engajam nas atividades orientadas para aprendizagem antes, durante e depois do treinamento. Há ainda outro estado afetivo presente ao final do treinamento que merece atenção: a extensão na qual os indivíduos estão motivados para aplicar o conteúdo que aprenderam. O objetivo principal de muitos cursos é aumentar a disposição do participante para usar, no trabalho, o material visto no programa, o que é denominado Motivação para Transferir.

Alguns pesquisadores como Noe e Schmitt (1986) e Colquitt e Simmering (1998) têm sugerido que os aspectos motivacionais relacionados ao treinamento deveriam ser examinados sob a perspectiva da teoria de expectância de Vroom (1964). Essa teoria é baseada na idéia de que o indivíduo formula expectativas cognitivas a respeito de efeitos decorrentes de seus próprios comportamentos e do valor relativo que atribui a cada um desses efeitos. Dessa maneira, o treinamento tem um valor particular para o indivíduo, porque serve como ferramenta ou instrumento para o alcance de outros resultados que ele espera e valoriza, como uma expectativa de promoção ou aumento de salário.

A teoria da expectância de Vroom (1964) é uma teoria cognitiva de processo, que trata de um modelo multiplicativo. Este modelo busca predizer comportamentos de escolha e sugere que a motivação de uma pessoa para tomar uma decisão é função dessas três variáveis: Valência versus Instrumentalidade versus 
Expectância. Os três componentes do modelo multiplicativo medem a força motivacional, mas são considerados construtos distintos.

A valência significa o quanto um indivíduo deseja uma recompensa ou uma escolha em relação a um resultado particular. A instrumentalidade diz respeito à estimativa de que determinado desempenho seja um caminho adequado para chegar a uma recompensa. Régis (2000) salienta que o desempenho pode estar relacionado, por exemplo, ao envolvimento com um programa de treinamento e não necessariamente a resultados relativos às suas tarefas no trabalho. Já a expectância se refere à estimativa de que o esforço de um indivíduo resultará em um desempenho bem-sucedido ou a chance de que esse desempenho produza o resultado esperado.

Neste trabalho, utilizou-se um conceito bidimensional de Valor Instrumental do Treinamento, abrangendo as definições de duas variáveis da teoria de expectância de Vroom (1964): Instrumentalidade e Valência. Dessa maneira, Valor Instrumental do Treinamento é definido como a crença do indivíduo de que as novas habilidades por ele adquiridas em um curso lhe serão úteis para atingir recompensas de várias naturezas (instrumentalidade), bem como a importância ou o valor que esse indivíduo atribui a cada uma dessas recompensas (valência). Portanto esse conceito diz respeito às escolhas particulares do indivíduo e aos benefícios do programa de treinamento para o treinando, relacionados a resultados futuros. Abbad, Pantoja e Pilati (2001, p.9), em sua recente revisão de literatura, analisaram artigos publicados entre os anos de 1998 e 2001 e observaram que "as pesquisas brasileiras e estrangeiras sobre características individuais, de forma geral, indicaram ainda que motivação para o treinamento está relacionada com os três níveis individuais de avaliação". Esses autores também observaram que as variáveis motivacionais mostraram-se mais fortes preditoras de sucesso individual em treinamento do que capacidades cognitivas e características demográficas da clientela, na amostra de artigos estrangeiros.

Embora a literatura em geral seja clara a respeito da influência de Motivação para Aprender sobre resultados de aprendizagem, há necessidade de tornar mais precisos alguns conceitos, conforme indicaram Salas e Cannon-Bowers (2001). Esses autores também afirmam que Motivação para Aprender, Auto-Eficácia, Aprendizagem e Transferência da Aprendizagem são as variáveis que necessitam de maior atenção dos pesquisadores nos próximos anos.

O presente estudo utilizará o conceito de Motivação para Aprender como o interesse do treinando em aprender o conteúdo do programa de treinamento e Motivação para Transferir como o grau de interesse do treinando em aplicar no trabalho os conteúdos aprendidos no treinamento. 
Em suma, os poucos dados empíricos disponíveis na literatura especializada mostram que os níveis de avaliação parecem depender de certas características motivacionais dos participantes de treinamentos. Além disso, na literatura revisada, há poucos investimentos na produção de instrumentos para os construtos de Motivação para Aprender, Motivação para Transferir e Valor Instrumental do Treinamento, entre eles os instrumentos produzidos por Noe e Schmitt (1986) e Abbad (1999).

\section{Contexto de Treinamento}

Outra linha de pesquisa da área de avaliação refere-se à influência exercida pelo contexto organizacional nos resultados de treinamento. Os trabalhos dessa linha investigam o relacionamento entre fatores ambientais e eficácia de programas de treinamento, ou seja, examinam se a eficácia de programas de treinamento pode ser influenciada por variáveis como suporte e clima organizacionais.

Para Abbad (1999) os termos Suporte, Clima para Transferência, Contexto Funcional e Ambiente têm sido usados como sinônimos, o que pode causar confusões teóricas e metodológicas para a área. Neste estudo, serão utilizados os conceitos de apoio ou suporte ao treinamento.

Um dos conceitos relativos ao ambiente organizacional é a percepção do apoio gerencial ao treinamento. Esse conceito, segundo Abbad (1999), refere-se à opinião do participante do treinamento a respeito do empenho demonstrado pelas chefias para viabilizar a sua participação em eventos instrucionais. Refere-se, portanto, a certas condições do ambiente pré-treinamento e ao nível de apoio recebido pelo treinando para participar efetivamente de todas as sessões de treinamento. Neste trabalho, será denominado Suporte Pré-Treinamento.

O segundo conceito referente à organização é o Suporte à Transferência de treinamento, que compreende suporte material e psicossocial (fatores situacionais de apoio e conseqüências associadas ao uso das novas habilidades) à aplicação no trabalho de novas habilidades. Alguns estudos indicam que variáveis psicossociais de apoio ao uso de novas habilidades no trabalho são importantes preditoras de Transferência de Treinamento, de acordo com Abbad (1999), Abbad, Pantoja e Pilati (2001) e Salas e Cannon-Bowers (2001). Roullier e Goldstein (1993), por exemplo, definiram, construíram e validaram uma medida de Clima, demonstrando que mais da metade da variabilidade da transferência de treinamento era explicada por variáveis relacionadas ao Clima para Transferência. O Suporte Psicossocial à Transferência ou Clima para Transferência também apareceu como forte preditor de Impacto do Treinamento no Trabalho em pesquisas nacionais (Abbad et al., 1999; Pantoja, Lima e Borges-Andrade, 1999). 
Borges-Andrade e Abbad (1996) verificaram que os empregados cujos chefes forneciam apoio para que participassem dos treinamentos, possuíam atitudes favoráveis em relação ao curso. Ações gerenciais, de colegas ou da própria política organizacional proporcionam sugestões e sinais que influenciam a motivação dos empregados. Supervisores podem demonstrar apoio a um treinamento por meio de discussões com o empregado, estabelecimento de metas de trabalho a partir do treinamento, liberação de tempo para preparação e encorajamento do trabalhador a engajar-se com o treinamento.

Para Abbad (1999) Suporte à Transferência de treinamento é um componente crítico no estudo das variáveis que afetam a eficácia dos eventos instrucionais. Estudos referentes a contextos de treinamento demonstram a influência das variáveis do nível gerencial e organizacional sobre a Transferência de Treinamento, Reação e Motivação para Aprender (ver Abbad, Pantoja e Pilati, 2001; Salas e Cannon-Bowers, 2001).

A análise da literatura apresentada neste estudo mostra que as variáveis motivacionais precisam de melhorias em suas medidas e a relação entre os níveis de avaliação ainda não é conclusiva. Além disso, são poucos os estudos que procuram identificar as variáveis explicativas de cada um desses níveis de avaliação. Os resultados das pesquisas analisadas sugerem ainda que variáveis motivacionais, tais como Motivação para Aprender, Motivação para Transferir e Valor Instrumental do Treinamento são correlacionadas positivamente com Impacto do Treinamento no Trabalho. Espera-se também que variáveis relativas à organização, como Suporte Pré-Treinamento e Suporte à Transferência, sejam preditoras da variável Impacto citada.

Abbad (1999) sugeriu várias providências para o aperfeiçoamento do Modelo Integrado de Avaliação de Impacto do Treinamento no Trabalho. Entre elas, pretende-se, neste trabalho, atender, em parte, às seguintes: (1) "definir motivação para o treinamento e para transferência e construir uma escala de avaliação desses construtos [e (2)] definir melhor o conceito de Valor Instrumental do Treinamento" (Abbad,1999, p. 250, 242). Essa autora também salientou que "é necessário analisar de que modo se articulam essas motivações com os planos profissionais do participante” (Abbad, 1999, p.242).

Além disso, Abbad (1999, p.236) sugeriu a diminuição do número de variáveis independentes nos modelos de regressão, "para garantir explicações mais parcimoniosas” e evitar as chances de erro Tipo I, que poderiam ocorrer no caso da inclusão de muitas "variáveis independentes nos modelos de regressão múltipla".

Foram definidos os seguintes objetivos para este trabalho, a partir do exame da literatura: (1) construir e validar a escala de Valor Instrumental do Treinamento; (2) aperfeiçoar e validar as escalas de Motivação para Aprender e Motivação 
para Transferir, adaptadas de Warr, Allan e Birdi (1999), e de Suporte PréTreinamento, adaptada da escala de apoio gerencial ao treinamento de Abbad (1999); e (3) investigar as variáveis preditoras de Impacto do Treinamento no Trabalho de cursos de uma organização pública do Distrito Federal, DF, no contexto de treinamentos presenciais, baseado principalmente no Modelo IMPACT de Abbad (1999). Nesse último objetivo, espera-se que Motivação para Aprender, Motivação para Transferir, Valor Instrumental do Treinamento, Suporte PréTreinamento, Suporte Psicossocial à Transferência, Suporte Material à Transferência, Reação ao Programa e Reação ao Desempenho do Instrutor expliquem uma porção significativa de variabilidade das respostas dos participantes à escala de Impacto do Treinamento no Trabalho.

\section{Método}

A presente pesquisa foi realizada no período de abril a outubro de 2001 no Instituto Serzedêllo Corrêa (ISC), órgão do Tribunal de Contas da União (TCU), sediado em Brasília, DF. Ao todo, foram avaliados 28 cursos oferecidos pelo ISC, perfazendo uma carga horária total de 635 horas.

O presente trabalho foi realizado em três etapas, conforme os objetivos citados anteriormente. A etapa 1 refere-se à construção e validação da escala de Valor Instrumental do Treinamento; a etapa 2 diz respeito ao aperfeiçoamento e validação das escalas de Motivação para Aprender, Motivação para Transferir e de Suporte Pré-Treinamento; e a última visa à investigação das variáveis preditoras de Impacto do Treinamento no Trabalho, de acordo com o modelo de avaliação proposto na Figura 1.

\section{Figura 1: Modelo de Lacerda}

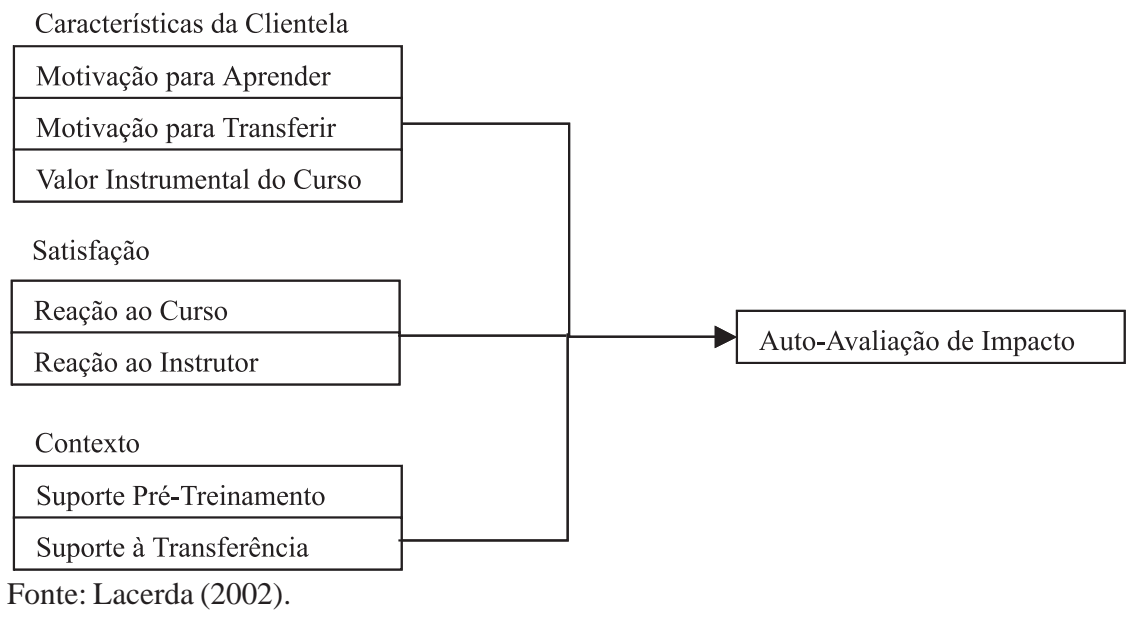




\section{Características da Amostra de Participantes}

Para a validação dos instrumentos, foi utilizada uma amostra com 284 casos, excluídos os casos extremos. Para a investigação das variáveis explicativas de Impacto do Treinamento no Trabalho (Etapa 3), foram utilizados 95 casos (sem os casos extremos). Os participantes desta última amostra tinham as seguintes características: $75,8 \%$ possuíam curso superior completo; $69,2 \%$ eram do sexo masculino; 64,1\% eram casados; tinham uma média de idade de 35,8 anos $(\mathrm{DP}=8,37)$ e um tempo médio de serviço no TCU de 7,0 anos $(\mathrm{DP}=6,3)$. Além disso, 69,2\% dos participantes ocupa cargo de nível superior.

\section{Instrumentos e Procedimentos de Coleta de Dados}

Foram aplicados quatro questionários em quatro momentos distintos: no início, durante, no final e após o treinamento, conforme indica o Quadro 1.

\section{Quadro 1: Momento de Aplicação e Instrumentos Utilizados}

\begin{tabular}{llll}
\hline Momento de Aplicação & Instrumentos de Avaliação & & \\
\hline No início do treinamento & Motivação para Aprender e Suporte & Pré- \\
& Treinamento & & \\
Durante o treinamento & Roteiro de Análise do material didático & \\
No final do treinamento & Avaliação do Curso \\
45 dias após o término do curso & Impacto do Treinamento no Trabalho \\
\hline
\end{tabular}

\section{Procedimentos de Análise de Dados}

Os dados coletados por meio dos questionários foram transcritos para um arquivo de dados. Neste arquivo, as respostas dos participantes aos questionários foram pareadas, de forma a possibilitar a análise do modelo de regressão múltipla. Para a execução das análises estatísticas, utilizou-se o Programa Estatístico SPSS (Statistical Package for Social Sciences), versão 10.0.

Foram realizadas análises descritivas e exploratórias, de modo a identificar os casos extremos e a distribuição de dados omissos. Para a identificação dos casos extremos multivariados utilizou-se o método da distância mahalanobis. Os casos extremos univariados foram localizados com a utilização de gráficos box plot. O critério para retirada dos casos multivariados foi: mahalanobis distance $<73,402$ ( $p<0,001)$, de acordo com a Tabela de Valores Críticos do Quiquadrado (Tabachnick e Fidell, 1996). Além disso, foram realizadas análises de componentes principais (PC), análises fatoriais (PAF) e de consistência interna (Alfa de 
Cronbach - $\alpha$ ), para validar as escalas. Análises de regressão múltipla padrão foram utilizadas para verificar as variáveis preditoras de Impacto do Treinamento no Trabalho. Para otimizar o aproveitamento dos casos, adotou-se o tratamento pairwise para os dados omissos em todas as análises.

\section{Resultados}

Na análise exploratória dos dados, após a localização dos casos extremos, foram utilizados os critérios para sua retirada, conforme citado anteriormente (37 univariados e 202 multivariados), o que resultou no aproveitamento de 284 casos para as etapas 1 e 2 e 95 casos para a terceira etapa. Além disso, o número de casos omissos não chegou a $5 \%$.

As análises fatoriais mostraram que as escalas estudadas na primeira etapa são unidimensionais, conforme descrição apresentada na Tabela 1.

Tabela 1: Estrutura Empírica das Escalas dos Estudos 1 e 2

\begin{tabular}{llllll}
\hline Escala & Fator & \% da variância & $\alpha$ & $\mathrm{n}^{\circ}$ de itens & carga \\
\hline Valor Instrumental & 1 & 37,62 & 0,94 & 27 & 0,32 a 0,73 \\
Motivação p/Aprender & 1 & 52,02 & 0,75 & 5 & 0,50 a 0,71 \\
Motivação p/ Transferir & 1 & 67,78 & 0,86 & 5 & 0,61 a 0,89 \\
Suporte Pré-Trein. & 1 & 58,01 & 0,85 & 6 & 0,47 a 0,82 \\
\hline
\end{tabular}

Todas as escalas contêm apenas um fator, uma vez que os valores próprios, a porcentagem da variância total explicada pelo segundo fator (menor do que $3 \%$, conforme critério de Harman, sugerido por Pasquali [2000]) e o teste do scree plot sugeriam a existência de estruturas unifatoriais para as 4 escalas.

A análise de regressão múltipla padrão mostrou-se a mais adequada para testar o modelo de avaliação de Impacto do Treinamento no Trabalho, de acordo com a orientação de Tabachnick e Fidell (1996). Essa técnica requer um número de respondentes por variável inferior ao exigido por outras estratégias como a regressão múltipla stepwise, por exemplo. Na primeira análise de regressão múltipla efetuada, houve sinais de redundância com o fator Suporte PréTreinamento. Dessa maneira, ele foi retirado do modelo, já que sua permanência demandaria análises mais complexas, o que fugiria ao escopo deste trabalho. Uma nova análise padrão foi efetuada sem essa variável. Os resultados da regressão múltipla padrão são apresentados na Tabela 2. 


\section{Tabela 2: Modelo de Regressão Múltipla para a Variável Critério Impacto do Treinamento no Trabalho}

\begin{tabular}{lllllllll}
\hline Variáveis & 1 & 2 & 3 & 4 & 5 & 6 & 7 & 8 \\
\hline 1. Impacto & & & & & & & & \\
2. Motivação para Aprender & 0,3 & & & & & & & \\
3. Valor Instrumental & 0,55 & 0,28 & & & & & & \\
4. Motivação para Transferir & 0,61 & 0,36 & 0,39 & & & & & \\
5. Suporte Psicossocial & 0,55 & 0,17 & 0,39 & 0,53 & & & & \\
6. Suporte Material & 0,07 & 0,06 & 0,24 & 0,15 & 0,34 & & & \\
7. Reação ao Programa & 0,62 & 0,26 & 0,5 & 0,68 & 0,36 & 0,14 & & \\
8. Reação ao Instrutor & 0,56 & 0,12 & 0,4 & 0,42 & 0,27 & 0,01 & 0,75 & \\
& & & & & & & & \\
Médias & 3,4 & 4,5 & 3,3 & 4,2 & 3,1 & 3,6 & 3,8 & 4,3 \\
Desvios-Padrões & 0,77 & 0,45 & 0,75 & 0,63 & 0,82 & 0,69 & 0,59 & 0,65 \\
B & & 0,13 & $0,23 * *$ & 0,25 & $0,28 * *$ & $-0,15$ & 0,09 & $0,29 *$ \\
$\beta$ & & 0,07 & 0,23 & 0,21 & 0,3 & $-0,14$ & 0,07 & 0,25 \\
Sr & & 0,07 & 0,19 & 0,13 & 0,23 & $-0,13$ & 0,03 & 0,16 \\
\hline Constante $=-0,93$ & $\mathrm{R}=0,78$ & $\mathrm{R}^{2}=0,61^{\mathrm{a}}$ & $\mathrm{R}^{2}$ (ajustado) $=0,58$ & \\
\hline
\end{tabular}

Nota: ${ }^{* *} \mathrm{p}<0,01 ;{ }^{*} \mathrm{p}<0,05 ;{ }^{\text {a }}$ Variabilidade singular $=0,58$; Variabilidade compartilhada $=0,03$.

A Tabela 2 demonstra que apenas três variáveis das sete incluídas na análise entraram na equação de regressão como preditoras de Impacto do Treinamento no Trabalho. São elas: Suporte Psicossocial $\left(\mathrm{Sr}^{2}=0,23\right)$, Valor Instrumental do Treinamento $\left(\mathrm{Sr}^{2}=0,19\right)$ e Reação ao Desempenho do Instrutor $\left(\mathrm{Sr}^{2}=0,16\right)$. Juntas, essas três variáveis explicam aproximadamente 61\% (58\% ajustado) da variabilidade no Impacto do Treinamento no Trabalho. Verifica-se que o valor da correlação múltipla (R) é alto e significativamente diferente de zero. Na Figura 2, o Modelo Preditivo de Impacto do Treinamento no Trabalho (45 dias após o término do treinamento) é esquematizado, apresentando as três variáveis explicativas de Impacto do Treinamento no Trabalho.

Figura 2: Modelo Obtido de Impacto do Treinamento no Trabalho

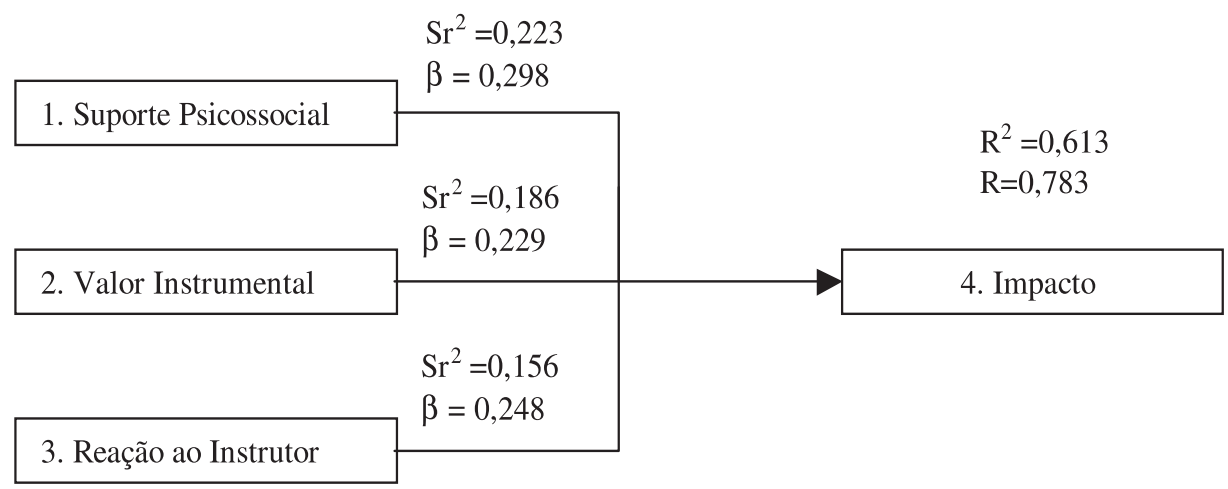




\section{Discussão}

Os resultados da etapa 1 demonstraram que a escala construída "Valor Instrumental do Treinamento" mostrou índices psicométricos adequados às exigências da área. Essa escala apresentou alto índice de consistência interna $(\alpha=0,94)$.

Assim uma importante implicação dos resultados dessa validação foi a melhoria da definição do conceito de Valor Instrumental do Treinamento, atendendo a uma solicitação de Abbad (1999). Colquitt, LePine e Noe (2000) abordaram apenas o conceito de valência em seu modelo da teoria integrativa de motivação para o treinamento. No presente trabalho, o termo passa a possuir maior abrangência em termos conceituais, envolvendo duas variáveis da Teoria da Expectância de Vroom (1964): valência e instrumentalidade de um indivíduo em relação a eventos de treinamento.

Dessa maneira, as teorias de processo sugeridas por Vroom (1964) demonstram que a motivação de uma pessoa com relação a determinado treinamento, é baseada na crença de que ela pode atingir resultados importantes, úteis e valorizados, melhorar sua atuação em outras tarefas, elevar suas chances de ascensão na carreira, aumentar sua empregabilidade ou atingir suas aspirações profissionais. Os conceitos de motivação para aprender e motivação para transferir, utilizados na literatura da área, limitam-se a investigar o interesse genérico do treinando em relação ao curso. Sugere-se, em futuros estudos, incluir nos modelos motivacionais de avaliação de treinamento o conceito de expectância ou expectativa apresentado por Vroom (1964), não utilizado neste trabalho, de modo a abranger os três conceitos propostos por ele: valência, instrumentalidade e expectância.

Uma importante questão prática deste estudo diz respeito à atribuição de conseqüências, pelos indivíduos, na participação em treinamentos por eles valorizados. Desta maneira, é relevante apresentar aos treinandos o valor dos programas instrucionais (que ganhos se pode obter deles) para que eles se sintam motivados a participar dos programas de treinamento. Programas não relacionados a resultados futuros, desejados pelo indivíduo, poderão não alcançar seus objetivos. Por essa razão, é necessário que os gestores da organização identifiquem os planos e as aspirações profissionais dos participantes de treinamentos, por exemplo, criando um banco de talentos ou competências e, com essas informações, viabilizando a programação de ações de educação continuada (oportunidades para que o trabalhador invista em sua formação acadêmica/profissional, evitando a obsolescência).

Com relação aos resultados obtidos na etapa 2, a escala de Motivação para 
Transferir obteve um índice de consistência interna de $\alpha=0,86$, maior que o encontrado por Warr, Allan e Birdi (1999): $\alpha=0,79$. A escala de Suporte PréTreinamento obteve $\alpha=0,85$, um pouco maior que o encontrado por Abbad (1999): $\alpha=0$,82. Essas escalas obtiveram melhoria razoável em relação às que as originaram, no que se refere às características psicométricas analisadas. Já a escala de Motivação para Aprender obteve $\alpha=0,75$, um pouco menor que o encontrado por Warr, Allan e Birdi (1999): $\alpha=0,76$.

Sugere-se, em futuras pesquisas, que seja dada maior atenção às variáveis de processo para a melhoria de escalas que envolvem conceitos motivacionais, conforme mencionado anteriormente, visto que as escalas de Motivação para Aprender e Motivação para Transferir necessitam ser aperfeiçoadas.

De modo geral, os instrumentos de medida construídos e validados por Abbad (1999), utilizados nesta pesquisa, mantêm-se estáveis e altamente confiáveis. Os resultados da terceira etapa indicaram que três variáveis são explicativas de Impacto do Treinamento no Trabalho: (1) Suporte Psicossocial; (2) Valor Instrumental do Treinamento; e (3) Reação ao Desempenho do Instrutor. Suporte Psicossocial, como forte variável preditora de Impacto do Treinamento no Trabalho, corrobora os achados da área (Roullier e Goldstein, 1993; Abbad et al., 1999; Pantoja, Lima e Borges-Andrade, 1999; Colquitt, Lepine e Noe, 2000; Salas e Cannon-Bowers, 2001).

Valor Instrumental do Treinamento, a segunda variável explicativa de Impacto do Treinamento no Trabalho no modelo deste estudo, apresentou resultado inédito na literatura. Seu poder explicativo talvez possa ser explicado por estar relacionado com a atual conjuntura de Treinamento e Desenvolvimento, na qual os trabalhadores se vêem diante de grandes exigências no mercado de trabalho, que busca colaboradores cada vez mais qualificados e preparados. Assim a motivação de um indivíduo para determinado curso e o seu conseqüente impacto no trabalho podem estar vinculados aos ganhos secundários associados ao treinamento, tal como medidos pela escala de Valor Instrumental.

A entrada de Valor Instrumental do Treinamento como uma variável motivacional explicativa de impacto no modelo estudado, corrobora resultados obtidos por pesquisas sobre a relação positiva entre motivação e resultados do treinamento (Warr e Bunce, 1995; Abbad, 1999; Colquitt, Lepine e Noe, 2000). É necessário que a variável Valor Instrumental do Treinamento seja investigada em futuras pesquisas, diversificando organizações, amostras de cursos e participantes, a fim de avaliar a generalidade dos resultados desta pesquisa.

As outras variáveis motivacionais utilizadas nesse estudo, Motivação para 
Aprender e Motivação para Transferir, não foram explicativas de Impacto do Treinamento no Trabalho, contrariando resultados de pesquisas nacionais e estrangeiras. Uma possível explicação para a exclusão dessas variáveis no modelo de predição de Impacto do Treinamento no Trabalho se refere a problemas de qualidade dos instrumentos de medida, que, conforme já citado, deverão ser aprimorados em estudos posteriores.

A terceira variável preditora de impacto, Reação ao Desempenho do Instrutor, corrobora os achados de Abbad (1999), Warr, Allan e Birdi (1999) e Abbad, Gama e Borges-Andrade (2000) e refuta os de Alliger et al. (1997).

Deve-se, entretanto, evitar generalizações desse resultado, pois a organização estudada nesta pesquisa foi a mesma utilizada nos trabalhos de Abbad (1999). Isso poderia indicar uma característica própria da organização, uma tendência dos servidores em considerar mais sua satisfação com o desempenho do instrutor que com outros aspectos do programa do treinamento, e esse fator influenciar no Impacto do Treinamento no Trabalho. Talvez isso possa explicar a não entrada da variável Reação ao Programa no modelo estudado. Por essa razão, é altamente indicado que o modelo de avaliação de Impacto do Treinamento no Trabalho proposto neste estudo, seja replicado por futuros pesquisadores em diferentes organizações para comparar seus resultados.

\section{Conclusões e Considerações Finais}

Os resultados obtidos com a variável Suporte Psicossocial como a melhor preditora de Impacto do Treinamento no Trabalho neste estudo, podem ser um indício de generalidade, conforme salientaram Abbad, Pantoja e Pilati (2001) e Salas e Cannon-Bowers (2001). Os resultados referentes ao poder explicativo de Valor Instrumental do Treinamento e Reação ao Instrutor sobre impacto ainda não são generalizáveis. Sugere-se, diante disto, a realização de novos estudos que os integrem como preditores da variável critério aqui tratada.

As contribuições mais relevantes deste trabalho referem-se à boa qualidade psicométrica da escala construída de Valor Instrumental do Treinamento e ao fato de essa variável ter sido explicativa de impacto. Acrescentam-se ainda as implicações teóricas e práticas decorrentes desses resultados, já citadas anteriormente.

As limitações desta pesquisa foram: (1) a restrição da amostra de participantes de uma organização apenas, o que reduz a generalidade dos resultados; (2) a impossibilidade de aplicação de questionários de heteroavaliação de Impacto do 
Treinamento no Trabalho, que poderiam gerar resultados comparativos com os de auto-avaliação; (3) a baixa devolução de questionários de Impacto do Treinamento no Trabalho e a não avaliação do padrão de não devolução; (4) a inexistência de pré e pós-testagem de habilidades treinadas para análise do relacionamento entre Aprendizagem, Reações e Impacto do Treinamento no Trabalho; (5) a falta de treinamentos destinados a desenvolver as habilidades psicomotoras; e (6) a pequena participação de treinandos com baixa escolaridade.

De acordo com os resultados apresentados e discutidos, propõe-se a seguinte agenda de pesquisa para futuros estudos: (1) aperfeiçoar e aplicar os instrumentos construídos e validados neste estudo em novas organizações, cursos e metodologias, em treinamentos presenciais e à distância, utilizando diferentes variáveis critério, de modo a confirmar sua confiabilidade e replicação; (2) utilizar medidas de impacto em profundidade, baseadas em objetivos de treinamento; (3) ampliar o modelo teórico proposto com a inclusão do nível Aprendizagem (pré e pós-testagem); (4) analisar a relação das variáveis Suporte Pré-Treinamento e Motivação para Aprender, de preferência utilizando análises mais complexas como equação estrutural, para investigar relacionamentos diretos e indiretos entre as variáveis estudadas; (5) verificar as Características da Clientela e os possíveis fatores associados aos participantes que não devolveram o questionário, de maneira a estudar estratégias para aumentar a participação das pessoas na devolução desse instrumento devidamente preenchido; (6) continuar investigando o poder preditivo das variáveis motivacionais, não somente no Impacto do Treinamento no Trabalho, mas em outros níveis de avaliação de resultados, como Reação e Aprendizagem; e (7) identificar a relação entre os três níveis de avaliação de resultados de treinamento, ainda não conclusiva na literatura.

\section{Nota}

${ }^{1}$ A primeira versão deste artigo foi premiada com menção honrosa no ENANPAD 2002.

\section{Referências Bibliográficas}

ABBAD, G.

Um modelo integrado de avaliação de impacto do treinamento no trabalho -
IMPACT. Brasília, 1999. Tese (Doutorado) - Instituto de Psicologia, Universidade de Brasília. 
ABBAD, G. et al.

Preditores de impacto do treinamento no trabalho - o caso do TCU. In: REUNIÃO ANUAL DE PSICOLOGIA, 29., 1999, Ribeirão Preto. Anais... Ribeirão Preto: Sociedade Brasileira de Psicologia, 1999. p. 53. Resumo.

ABBAD, G.;

GAMA, A. L. G.;

BORGES-ANDRADE, J. E.

Treinamento: análise do relacionamento da avaliação nos níveis de reação, aprendizagem e impacto no trabalho. Revista de Administração Contemporânea, v. 4, n. 3, p. 25-45, 2000.

ABBAD, G.;

PANTOJA, M. J.;

PILATI, R.

Avaliação de treinamento: o estado da arte e o futuro necessário. In: ENCONTRO ANUAL DA ANPAD, 25., 2001, Campinas. Anais... Rio de Janeiro: ANPAD, 2001. 1 CDROM.

ALLIGER, G. M. et al.

A meta-analysis of the relations among training criteria.

Personnel Psychology, v. 50, p. 341-358, 1997.

ALLIGER, G. M.;

JANAK, E. A.

Kirkpatrick's levels of training criteria: thirty years later. Personnel Psychology, v. 42, n. 2, p. 331-342, 1989.
BORGES-ANDRADE, J. E.

Avaliação somativa de sistemas instrucionais: integração de três propostas. Tecnologia

Educacional, v. 11, n. 46, p. 2939, 1982.

Desenvolvimento de medidas em avaliação de treinamento. In: ENCONTRO ANUAL DA ANPAD, 24., 2000, Florianópolis. Anais... Rio de Janeiro: ANPAD, 2000. 1 CD-ROM.

BORGES-ANDRADE, J. E;

ABBAD, G.

Treinamento no Brasil: reflexões sobre suas pesquisas. Revista de Administração, v. 31, n. 2, p. 112125, 1996.

COLQUITT, J. A.;

LEPINE, J. A.;

NOE, R. N.

Toward an integrative theory of training motivation: a meta-analytic path analysis of 20 years of research. Journal of Applied Psychology, v. 85, n. 5, p. 678707, 2000.

COLQUITT, J. A.;

SIMMERING, M. J.

Conscientiousness, goal orientation and motivation to learn during the learning process: a longitudinal study. Journal of Applied Psychology, v. 83, n. 4, p. 654665, 1998. 
GOLDSTEIN, I. L.

Training in work organizations. In: DUNNET; HOUGH (Orgs). Handbook of industrial and organizational psychology. 2. ed. California: Consulting Psychology Press, 1991. p. 507-619.

HAMBLIN, A. C.

Avaliação e controle de treinamento. São Paulo: McGraw-Hill, 1978.

KIRKPATRICK, D. L.

Evaluation of training. In: CRAIG, R. L. Training and development handbook. 2. ed. New York: McGraw-Hill, 1976. p. 18.1-18.27.

\section{LACERDA, E. R. M.}

Impacto do treinamento no trabalho: investigando variáveis motivacionais e organizacionais como suas preditoras. Brasília, 2002. Dissertação (Mestrado) Instituto de Psicologia, Universidade de Brasília.

NOE, R. A.;

SCHMITT, N.

The influence of trainee attitudes on training effectiveness: test of a model. Personnel Psychology, v. 39, p. 497-523, 1986.

PANTOJA, M. J.;

LIMA, S .M. V.;

BORGES-ANDRADE, J. E.

Avaliação de impacto de treinamento na área de reabilitação: preditores individuais e situacionais. In: ENCONTRO ANUAL DA ANPAD, 23., 1999, Foz do Iguaçu. Anais... Rio de Janeiro: ANPAD, 1999. 1 CDROM.

PASQUALI, L.

Análise fatorial para pesquisadores. Brasília: LabPAM, 2000.

RÉGIS, H. P.

A motivação dos professores do Centro Federal de Educação Tecnológica da Paraíba para participarem do programa de qualidade: um exame com base na teoria da expectância. Recife, 2000. Dissertação (Mestrado) - Centro de Ciências Sociais Aplicadas, Universidade Federal de Pernambuco.

ROULLIER, J. Z.; GOLDSTEIN, I. L.

The relationship between organizational transfer climate and positive transfer of training. Human Resource Development Quarterly, v. 4, n. 4, p. 377-390, 1993.

\section{SALAS, E.;}

CANNON-BOWERS, J.

The science of training: a decade of progress. Annual Review of Psychology, v. 52, p. 471-499, 2001. 
TABACHNICK, B. G.;

FIDELL, L. S.

Using multivariate statistics. 3 . ed. New York: Harper \& Row, 1996.

VROOM, V. H.

Work and motivation. New York: John Wiley \& Sons, 1964.

WARR, P.;

ALLAN, C.;

BIRDI, K.

Predicting three levels of training outcome. Journal of Occupational and Organizational Psychology, v. 72, n. 3, p. 351-375, 1999.

WARR, P.;

BUNCE, D.

Trainee characteristics and outcomes of open learning. Personnel Psychology, v. 48, p. 347-375, 1995.

WEXLEY, K. N.

Personnel training. Annual Review of Psychology, v. 35, p. 519-51, 1984. 